\title{
Luminance controls the perceived 3-D structure of dynamic 2-D displays
}

\author{
BARRY J. SCHWARTZ and GEORGE SPERLING \\ New York University, New York, New York
}

\begin{abstract}
The accurate three-dimensional (3-D) appearance of two-dimensional (2-D) shadow projections cast by rotating 3-D objects-the kinetic depth effect (KDE)-frequently is used to visualize depth in computer-generated displays representing complex structures. According to current perceptual theories, the addition of simulated linear perspective will prevent illusory reversals of perspective associated with the $\mathrm{KDE}$, due to the geometric fact that the reversed-perspective percept is inconsistent with rigid object rotation. However, the reversed, nonrigid perceptual state can be induced by correlating luminance with the represented nearness of luminous lines, thus overriding geometric cues of form and motion that are supposed to govern the perception of rigid objects in the KDE. This finding complicates theories of 3-D motion perception based on 2-D arrays, but is of practical value in the design of visual displays representing complex 3-D objects.
\end{abstract}

The static two-dimensional (2-D) shadow projection of a wire cube-a cube made up of wire edges (Figure 1a)-can produce two radically different threedimensional (3-D) perceptions: One is the veridical, normal percept, and the other is an illusion in which the rear side appears to be in front-a reversed percept (Attneave, 1971; Gregory, 1970; Koffka, 1935). When a wire cube is depicted in rotation around a vertical axis, the perceptual alternation of 3-D form entails a corresponding change in the perceived direction of rotation. That is, if the veridically perceived 3-D cube appears to rotate in the clockwise direction, then the reversed figure appears to rotate in a counterclockwise direction. When the cube is viewed from a close range so that linear perspective is significant (Figures $1 \mathrm{~b}, 1 \mathrm{c}$, and $1 \mathrm{~d}$ ), then it alternates perceptually from a 3-D rigid cube to a nonrigid, distorting, rubbery-looking, unsymmetrical 3-D object.

When we depict such wire objects on a CRT screen, alternating rigid and nonrigid object perceptions are so compelling that naive subjects find it difficult to believe that the stimulus has not been altered physically as they watch. The alternation phenomenon is equally compelling with rotating random wire figures, even though these figures may appear flat when they are not rotating (Figures $1 \mathrm{e}$ and $1 \mathrm{i})$. When binocular disparity cues are provided, still figures (and, of course, rotating figures, too)

We wish to thank Charles S. Harris of Bell Laboratories for his collaboration in early demonstrations of nonrigidity in KDE figures with polar projection, and Thomas Riedl for his helpful advice on implementing proximity luminance covariances. This research was supported by NIMH Predoctoral Fellowship 5 F31 MH08172, and by U.S. Air Force, Life Sciences Directorate, Grant AF0SR-80-0279. The authors' mailing address is: Human Information Processing Laboratory, Department of Psychology, New York University, New York, New York 10003. are seen in vivid depth (stereopsis). However, we have observed that the illusion of 3-D depth in our rotating objects, monocularly viewed, is already so compelling that they do not differ discriminably from the same objects viewed binocularly with full stereopsis.

These peculiar examples of ambiguous perception represent instances of the "kinetic depth effect" (KDE), the phenomenon in which the 2-D shadow cast by a rotating 3-D wire object takes on the appearance of the projected object's motion and shape in $3 \mathrm{D}$, even in cases in which a static picture of the same object appears flat (Braunstein, 1976; Wallach \& O'Connell, 1953).

Theories of the KDE concern themselves with the process by which the visual system establishes the veridical, rigid 3-D perceptual structure from the geometric information in the changing 2-D picture (Bell \& Lappin, 1973; Börjesson \& von Hofsten, 1972; Gibson, 1966; Hay, 1966; Hershberger, 1967; Johansson, 1973; Todd, 1982; Ullman, 1979). Classical and current theories make no provision for the visual system's supplying a nonrigid perceptual interpretation when a rigid object interpretation is possible.

In this experiment, we show a class of simple wire stimuli in which nonrigid perceptions dominate overwhelmingly. This demonstration not only requires a revision of theories of the $\mathrm{KDE}$, but also is of practical importance to designers of visual displays.

The new display involves a depth-luminance covariance, or, in this instance, a proximity-luminance covariance (PLC). The luminance of each line in the 2-D projection of the object was made to depend on the 3-D depth of that line. The luminance varied from a maximum of $3.2 \mathrm{~cd} / \mathrm{m}$ luminous directional energy to a minimum value of $0.04 \mathrm{~cd} / \mathrm{m}(1.2 \%$ of the maximum) seen against a background of about $0.06 \mathrm{~cd} / \mathrm{m}^{2}$ (Sperling, 1971). That is, as the object rotated, the lines 

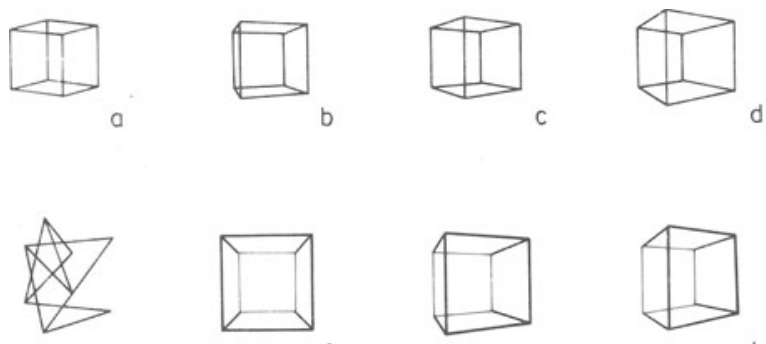

e
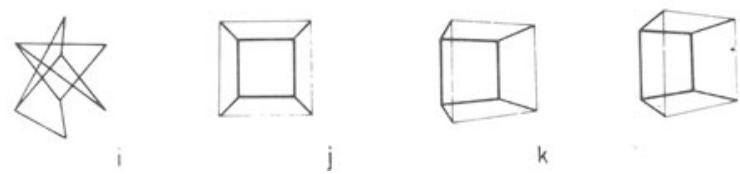

Figure 1. Line-drawing simulations depicting freeze-frame examples of experimental rotating stimuli. (a) Necker cube: a wire-cube 2-D projection drawn without linear perspective. (b-d) Wire cubes drawn with linear perspectives that simulate viewing real cubes from distances of $3 \times, 2.25 \times$, and $1.25 \times$ the width of a side, respectively. (e and $i)$ Two views of the same random-wire figure, drawn without linear perspective. (f-h) Three successive views of a cube with linear perspective as in (c), rotating counterclockwise from the top, displayed with a positive PLC. (j-1) Three successive views of the same linear perspective cube rotating counterclockwise from the top, displayed with a negative PLC.

in $3 \mathrm{D}$ nearest the observer were made to grow brighter or dimmer. Some manufacturers of display hardware offer PLC as an option in visual displays, ${ }^{1}$ but, to our knowledge, the effectiveness of this cue to depth has never before been investigated experimentally.

We investigated three different PLCs: first, a positive PLC in which the objectively near lines of the 3-D object are brightest and the furthest are dimmest (Figures $1 \mathrm{f}, 1 \mathrm{~g}$, and $1 \mathrm{~h}$ ). This cue simulates the effect on the retina of luminous thin lines of a rotating, rigid object as the lines approach and recede from the eye. Second, we investigated a negative PLC, one in which the more distant part of the 3-D cube is intensified (Figures 1j, 1k, and 11), the nonrigid percept being favored. Third, we investigated a neutral PLC condition, in which all lines were displayed with equal brightness (Figures 1a, 1b, 1c, and 1d).

The specific question tested experimentally was: How strongly do PLCs influence the perception of rotating wire figures. We examined figures displayed with different amounts of linear perspective. Subjects judged the rotation direction of each of a number of $\mathrm{KDE}$ stimuli. The judged direction is perfectly correlated with the perceptual alternative and thus indicates whether the object was perceived veridically (rigidly) or nonveridically (nonrigidly). The subjects viewed each stimulus and responded with a judgment of rotation direction as quickly as possible, usually within $3 \mathrm{sec}$. The subjects were asked only for their initial response to each stimulus. This response terminated the stimulus presentation and initiated the next stimulus, after a $2-\mathrm{sec}$ delay. In this way, all the different stimulus conditions were viewed in rapid succession.

All stimuli in this experiment consisted of perspective projections, on a CRT screen, of luminous cubes rotating around a vertical axis at a rate of 1 full revolution per $5 \mathrm{sec}$. Three degrees of polar projection were investigated, in addition to parallel projection, which carries no linear perspective foreshortening information. Polar projection is defined by the ratio of the largest projected side to the smallest projected side of the cube seen face on. Three values of polar projection ratio are: $1.40 / 1,1.57 / 1$, and $2.33 / 1$, the latter having the most extreme cue of linear perspective foreshortening. With parallel projection, the projection ratio is $1 / 1$. The actual size of objects on the CRT screen was the same in all conditions, about $3 \mathrm{~cm}$ at a viewing distance of $200 \mathrm{~cm}$. The two directions of rotation occurred equally often.

The principal result is an overwhelming effect of proximity luminance covariance (PLC) across all conditions of perspective. Table 1 shows results for $3 \mathrm{ob}$ servers who served as subjects for all the conditions; their data are representative of more than 20 observers who have viewed these displays. Each entry is based on at least 80 trials and represents the percentage of trials in which the observer correctly perceived the 3-D rigid body represented by the 2-D display. (On the remaining trials, the oppositely rotating, nonrigid alternative was perceived).

In stimuli with positive PLC, percepts of rigid object rotation predominated over nonrigid percepts $(96.5 \%$ to $4.1 \%)$. Negative PLC resulted in nonrigid percepts in an overwhelming majority of trials, regardless of polar perspective. With a negative PLC, the perspective and PLC conflict: The PLC wins on $90.5 \%$ of the trials (averaged over all polar-projection conditions).

The neutral (PLCO) conditions enable us to estimate the effectiveness of perspective alone as a determiner of perceived 3-D form. Clearly, perspective influences perceived 3-D form, but the effect is neither strong nor

Table 1

Effect of PLC on Dominarice of Rigid Object Rotation Averaged Over Polar Projection Conditions

\begin{tabular}{|c|c|c|c|c|c|}
\hline \multirow[b]{2}{*}{ Subject } & \multicolumn{3}{|c|}{ Percent Rigid Rotation } & \multirow{2}{*}{$\begin{array}{c}\text { Mean } \\
\text { (Subject) }\end{array}$} & \multirow{2}{*}{$\begin{array}{l}\text { Number } \\
\text { of Trials }\end{array}$} \\
\hline & PLC+ & PLC0 & PLC- & & \\
\hline $\begin{array}{l}\text { C.C. } \\
\text { N.B. } \\
\text { S.M. }\end{array}$ & $\begin{array}{l}99.3 \\
90.1 \\
100\end{array}$ & $\begin{array}{l}62.8 \\
44.2 \\
76.7\end{array}$ & $\begin{array}{r}10.1 \\
17.3 \\
1.1\end{array}$ & $\begin{array}{l}57.4 \\
50.5 \\
59.2\end{array}$ & $\begin{array}{l}576 \\
504 \\
648\end{array}$ \\
\hline Mean & 96.5 & 61.2 & 9.5 & 55.7 & 1728 \\
\hline
\end{tabular}

Note-Comparisons for three subjects include two conditions of presentation: rotation with and without static preview. The total number of judgments per subject in each PLC condition represents the number of sessions $x$ number of repetitions per session $x 3$ polar projections $x 2$ rotation directions $x 2$ types of experimental trial (with and without prior static context). 
consistent, with some subjects tending to see the rigid 3-D perceptual alternative and others the nonrigid alternative. In contrast, in parallel projection, in which perspective does not enable discrimination between alternatives, PLC determines the perceived direction of rotation on $97.4 \%$ of trials. Note that PLC influences which alternative is perceived but does not affect its shape or motion.

In a related experiment, we observed that when subjects viewed a static image with PLC, the perceptual alternative sometimes switched after the image started to rotate. The determination of the alternative perceptual states in moving stimuli was somewhat different from that in static stimuli, although PLC exerted a powerful influence on perceived depth in both kinds of stimuli. It should be noted that luminance per se is not the operative cue: In black-on-white wire figures, it is blackness that indicates nearness, suggesting that contrast (or some similar index of visibility or distinctness) is what signals "nearness" to the perceptual system.

Many other controls and variations in procedure were investigated but need not be detailed here because, unlike the stimuli, the results were completely unambiguous. The following experimental variations produced no qualitative difference in results: location of fixation point (within $10 \mathrm{deg}$ of the figure); distance from the observer to screen (from $20 \mathrm{~cm}$ to $3 \mathrm{~m}$ ); size of figure on screen (from1- to 5 -cm width); tilt of figure; monocular and binocular viewing (without stereopsis cues); speed of rotation (from 1 to $10 \mathrm{sec} /$ rotation); background illumination (darkness or normal room lighting); presence of other rotating figures on the screen; and even presence of conflicting stereopsis cues.

What do these results imply about how depth is perceived? Clearly, the interpretation of 3-D structure from a 2-D display is not based simply on a global reconstruction of the depth information inherent in perspective transformations: Perspective was a weak and unreliable cue. We propose instead that the 3-D perception is based on local cues-in these experiments, brightness, velocity, and the orientation of vertices, and in other experiments, interposition, texture gradients, and myriad other cues-and that these local cues are combined into a consistent global stereoptic image by a competition/cooperation interaction of the form proposed for stereopsis by Sperling $(1970,1981)$ and others (Grossberg, 1978; Hochberg, 1964; Marr, 1982). More experimentation is needed to establish this theoretical alternative. For the present, we offer practical advice for display designers who wish to make 2-D line displays appear like 3-D objects: Use both motion-in-depth (KDE) and a PLC to convey 3-D information. Perspective transformations may help some subjects but hinder others. In static as well as in moving representations, PLC is a powerful cue for disambiguating depth.

\section{REFERENCES}

Atrneave, F. Multistability in perception. Scientific American, 1971, 225(12), 62-71.

Bell, H. H., \& LAppin, J. S. Sufficient conditions for the discrimination of motion. Perception \& Psychophysics, 1973, 14, 45-50.

Böjesson, E., \& von Hofsten, C. Spatial determinants of depth perception in two-dot motion patterns. Perception \& Psychophysics, 1972, 11, 263-268.

Braunste in, M. L. Depth perception through motion. New York: Academic Press, 1976.

Gibson, J. J. The senses considered as a perceptual system. Boston: Houghton-Mifflin, 1966.

Gregory, R. L. The intelligent eye. London: Weidenfeld \& Nicholson, 1970.

Grossberg, S. Competition, decision, and consensus. Journal of Mathematical analysis and applications. 1978, 66, 470-493.

HAY, J. C. Optical motions and space perception: An extension of Gibson's analysis. Psychological Review, 1966, 73, 550-565.

Hershberger, W. A. Comment on the "apparent reversal (oscillation) of rotary motion in depth." Psychological Review, 1967, 74, 235-238.

Hochberg, J. E. Perception. Englewood Cliffs, N.J: PrenticeHall, 1964.

Johansson, G. Visual motion perception. Scientific American, 1973, 232(6), 76-88.

KoffKa, K. Principles of Gestalt psychology. New York: Harcourt, Brace \& World, 1935.

MARR, D. Vision: A computational investigation into the human representation and processing of visual information. San Francisco: Freeman, 1982.

Sperling, G. Binocular vision: A physical and neural theory. American Journal of Psychology, 1970, 83, 461-534.

Sperling, G. The description and luminous calibration of cathode ray oscilloscope visual displays. Behavior Research Methods \& Instrumentation, 1971, 3, 148-151.

Sperling, G. Mathematical models of binocular vision. In S. Grossberg (Ed.), Mathematical psychology and psychophysiology. Providence, R.I: Society of Industrial and Applied Mathematics-American Mathematical Association (SIAM-AMS) Proceedings, 1981, 13, 281-300.

ToDD, J . T. Visual information about rigid and nonrigid motion: A geometric analysis. Journal of Experimental Psychology: Human Perception and Performance, 1982, 8, 238-252.

Ullman, S. The interpretation of visual motion. Cambridge, Mass: MIT Press, 1979.

Wallach, H., \& O'Connell, D. N. The kinetic depth effect. Journal of Experimental Psychology, 1953, 45, 205-217.

\section{NOTE}

1. Picture System 2 (Evans \& Sutherland, Inc.), for example, incorporates "depth cuing"-hardware PLC.

(Manuscript recieved for publication October 5, 1983.) 